\title{
Los pianos de Liszt en Viena (1838-1846): un estudio de los instrumentos a través de artículos de prensa de la época
}

\author{
Liszt's Pianos in Vienna (1838-1846): \\ A Study of the Instruments through \\ Press Articles from the Period
}

Entre 1838 y 1846, varios críticos vieneses publicaron artículos sobre los conciertos que ofreció Liszt en la capital austríaca. Esos prolijos textos no solo contienen el comentario a la parte meramente interpretativa de la ejecución del programa, sino también numerosos detalles acerca de los pianos que utilizó el maestro húngaro en cada ocasión, las obras que tocó en ellos y el comportamiento de dichos instrumentos en el escenario.

El análisis de los documentos ha permitido conocer las características de los pianos, las marcas empleadas y cómo le asignaba Liszt las piezas a cada ejemplar. Los resultados han arrojado luz sobre el concepto sonoro del compositor en esos años -facilitando al intérprete de hoy en día la elección de instrumento para ese repertorio-, la actitud de la crítica respecto al medio sonoro concreto y la variedad de diseño de los pianos de aquel entonces.

Palabras clave: Liszt, piano, fortepiano, práctica interpretativa, Romanticismo.

Between 1838 and 1846, various Viennese critics published articles on the concerts Liszt gave in the Austrian capital. These meticulous texts not only contain comments on the merely interpretative aspects of the execution of the programs, but many other details, including the pianos the Hungarian composer used on each occasion, the works he played on them and way in which they functioned on stage.

An analysis of these reviews has revealed the characteristics of the pianos, their makers and how Liszt assigned the pieces to each of them. The results have shed some light on how the composer conceived sound during this period-thus making it easier for performers to choose an instrument for this repertory today-, the attitude of critics towards sound media and the variety of design of pianos built at the time.

Keywords: Liszt, piano, fortepiano, performance practice, Romanticism. 
La única observación que podría hacer es relativa al toque algo pesado y, sobre todo, con demasiado calado para mí; [...] porque me siento singularmente mimado con esas cómodas y agradables pantuflas ${ }^{1}$ de Viena, como las llamas de buena gana.

Carta de Liszt a Pierre Erard, 5-8-1847².

\section{Introducción}

Aunque numerosos trabajos de investigación sobre Liszt se han centrado en muchos aspectos de su vida y obra, el campo referido a los pianos que utilizó en cada época -tanto en el escenario como en el hogar- necesita aún de un examen profundo. Las series vienesas de conciertos de Liszt son particularmente interesantes para estudiar la relación del maestro con los sistemas constructivos del piano de finales de la primera mitad del siglo XIX, ya que en ellos tuvo, por primera vez, la oportunidad de elegir entre diferentes mecánicas en el escenario (en alguna ocasión, disponiendo incluso de instrumentos de tipos distintos simultáneamente). Esto era algo que no había ocurrido nunca hasta la primera de esas actuaciones públicas, que tuvo lugar en el 18 de abril de 1838, ya que, hasta entonces, había debido limitarse a emplear instrumentos de similar construcción en cada evento.

El único trabajo publicado que da una lista relativamente larga de los pianos que usó Liszt es un artículo de Geraldine Keeling de $1986^{3}$. En él, la autora, indudablemente limitada por las restricciones de espacio que suele tener un escrito de ese tipo, solo dedica tres párrafos en total al período y ámbito geográfico tratados en el presente estudio ${ }^{4}$. De la misma Keeling es también un artículo del mismo año que describe la relación de Liszt con el fabricante de pianos Johann Baptist Streicher. ${ }^{5}$ Aquí consigue examinar con más profundidad el fenómeno, dedicándole cinco páginas, pero el texto está centrado especialmente en los pianos del mencionado Streicher, de modo que el resto de marcas se nombra de manera colateral ${ }^{6}$. Si bien ambas investigaciones están bien documentadas y

\footnotetext{
${ }^{1}$ Con "pantuflas", Liszt se refiere a los pianos vieneses, de toque blando y ligero.

${ }^{2}$ Carta publicada en Franz Liszt, Michael Saffle, Michael Short (eds.): Liszt Letters in the Library of Congress, Hillsdale (NY), Pendragon Press, 2003, pp. 46 y 274. Traducción de la autora de este artículo.

${ }^{3}$ Geraldine Keeling: "The Liszt Pianos-Some Aspects of Preference and Technology", The New Hungarian Quarterly, 104, 1986, pp. 220-232.

${ }^{4}$ G. Keeling: "The Liszt Pianos...", pp. 221-222 y 225.

${ }^{5}$ Geraldine Keeling: "Liszt and J. B. Streicher, a Viennese Piano Maker", Studia Musicologia Academiae Scientiarum Hungaricae, 28, 1/4, 1986, pp. 35-46.

${ }^{6}$ G. Keeling: "Liszt and J. B. Streicher...", pp. 36-41.
} 
contienen información muy valiosa, ninguna de ellas se ocupa de investigar cuestiones como la relación entre obras e instrumentos concretos, así como el ideal sonoro lisztiano que de ella se traduce.

El principal objetivo de este estudio es determinar qué pianos utilizó Liszt en cada uno de los conciertos que ofreció en Viena en esos años, para saber quiénes fueron sus fabricantes y qué tipo de mecánica contenían. También se examinará cómo se comportaron los instrumentos en el escenario en lo que respecta a la estabilidad de la afinación, ruptura de cuerdas, potencia sonora, etc. Se especificará -en la medida en que sea posible- las obras que se tocaron en cada piano, con autores y títulos, lo cual llevará a comprobar si es posible establecer una relación entre repertorio e instrumentos concretos. Los resultados indicarán pautas para orientar al intérprete de hoy en día en la elección de piano histórico o en la adaptación al instrumento moderno cuando aborde el repertorio lisztiano de los últimos años del virtuoso ${ }^{7}$.

\section{Fuentes}

Para este estudio, se han empleado treinta y seis textos procedentes de tres periódicos vieneses de la época: el Allgemeine Theaterzeitung und Originalblatt für Kunst, Literatur, Musik, Mode und geselliges Leben ("Periódico general de teatro y hoja original de arte, literatura, música, moda y vida social"), el Wiener Allgemeine Musik-Zeitung" ("Periódico de música general vienés") y el Wiener Zeitung $^{10}$ ("Periódico vienés"). Todos los artículos recogidos son críticas, excepto una contribución de carácter humorístico de Franz Wiest, publicada el 11 de marzo de 1838 en el $A T h Z$.

El primero de los tres periódicos, el Allgemeine Theatherzeitung fue el más importante de su tiempo en el Imperio Austríaco en lo que a información sobre el panorama cultural se refiere. Comenzó su andadura en 1806 y cerró en 1860. Publicó numerosas críticas y noticias sobre música, demostrando estar muy pendiente de la actualidad del momento. El segundo, el Wiener Allgemeine Musik-Zeitung, tuvo una vida bastante corta (1841-1848), pero intensa. Como su título indica, estaba centrado únicamente en música. Sus corresponsales informaban no solo sobre eventos que tenían lugar en Viena, sino también en otras ciudades (por ejemplo, recogen noticias sobre conciertos de Liszt en Brno

\footnotetext{
${ }^{7}$ La vida de Liszt se divide tradicionalmente en tres épocas: la primera (1811-1847) comprende la infancia y los años de virtuoso; la segunda (1848-1861) es la llamada "de Weimar"; la tercera y última (1861-1886) se denomina simplemente como "de los últimos años" y contiene dos tramos, que son los años de Roma (1861-1869) y la "vida trifurcada" (1869-1886). Dicha división es de uso universal en la práctica totalidad del campo de la investigación sobre Liszt.

${ }^{8}$ Wiener Allgemeine Theaterzeitung, Viena, Geistinger, 1806-1860. En adelante, AThZ.

${ }^{9}$ Wiener Allgemeine Musik-Zeitung, Viena, Meccheti, 1841-1848. En adelante, WAMZ.

${ }^{10}$ Wiener Zeitung, Viena, van Ghelen'sche Erben, 1780-actualidad. En adelante, WZ.
} 
o Pest). El tercero, el Wiener Zeitung, fue fundado en 1703 y es el diario más antiguo del mundo aún en circulación. No es un periódico especializado en manifestaciones culturales, sino generalista.

Una figura esencial para este estudio es Heinrich Adami, autor de treinta de los documentos y crítico musical del Allgemeine Theaterzeitung durante dieciséis años, además de escritor de libros y adaptador de libretos de ópera ${ }^{11}$. Sus críticas son, en comparación con lo que suelen ser los escritos de ese tipo, excepcionalmente detalladas y extensas. En ellas, hay espacio para las reflexiones de tipo estético, para las comparaciones con otros virtuosos del panorama-como Thalberg o Clara Wieck- y para la descripción de las cualidades del pianismo lisztiano. También se comentan de manera exhaustiva las piezas interpretadas en cada actuación y, en la mayoría de los casos, se especifican los instrumentos utilizados en cada ocasión y su comportamiento en escena.

De los seis textos restantes, uno es obra de alguien que firmó con las iniciales "A. S.", que probablemente se corresponden con el nombre de August Schmidt, director y editor del WAMZ, que era, además de escritor y periodista, violinista, cantante y compositor ${ }^{12}$. Otro de ellos, aparecido en este mismo periódico, lo realizó un misterioso "Dr. K.", de identidad desconocida. Un tercero, de tintes humorísticos, fue redactado por Franz Wiest para el $A T h Z^{13}$. El cuarto es un artículo de Carl Walter para el $W Z^{14}$. Hay, además, dos anónimos (del $W A M Z$ y del $A T h Z$, respectivamente).

${ }^{11}$ Nacido en Viena en una familia burguesa, Adami (1807-1895) estudió derecho en la universidad de su ciudad natal, donde se graduó en 1829. En 1832 pasó a trabajar como colaborador fijo para el AThZ, puesto que ocupó ininterrumpidamente durante dieciséis años, ocupándose preferentemente de la crítica musical. Durante ese tiempo publicó, además, cuadernos y libros de diversos géneros y realizó adaptaciones de libretos de ópera y oratorio. Más tarde, dejó el ámbito de lo artístico y pasó a ser redactor del Wiener Zeitung, del Presse, y del Ostdeutsche-Post, en los que trató asuntos relacionados con el mundo jurídico y la política (Constant von Wurzbach: Biographisches Lexikon des kaiserthums Oesterreich, enthaltend die Lebensskizzen der denkwürdigen Personen, welche seit 1750 in den österreichischen Kronländer geboren wurden oder darin gelebt und gewirkt haben, Viena, K. K. Hof- und Staatsdrückerei, 1856-1891, vol. 1, p. 5).

${ }^{12}$ August Schmidt (1808-1891) nació en Viena. Su padre era un músico aficionado de buen nivel. Estudió violín y ofreció conciertos con ese instrumento. Poseía una bella voz, la cual le posibilitó convertirse también en cantante. Su paso por la vida militar le empujó a emprender una actividad compositiva muy fructífera. Escribió numerosos libros y artículos, muchos de los cuales trataban sobre música, y desempeñó un papel crucial en la fundación de diversas asociaciones musicales en su ciudad natal (C. von Wurzbach: Biographisches Lexikon..., 1875, vol. 30, pp. 219-228).

${ }^{13}$ Franz Wiest (1814-1847) recibió educación musical en su infancia. Estudió filosofía en la universidad y trabajó posteriormente como escritor, crítico y redactor. Colaboró con diversas revistas vienesas, en las que publicó, entre otros géneros, poemas y artículos de humor. Fue también dramaturgo y conferenciante humorístico. Su hermano Ludwig fue violín solista y director del Nemzeti Színház (Teatro Nacional) de Pest.

${ }^{14}$ Carl Walter era un colaborador habitual del Wiener Zeitung, autor de reseñas y críticas de teatro y música. 
Tabla 1. Identificación de los textos periodísticos consultados

\begin{tabular}{|c|c|c|c|c|}
\hline Fecha & Periódico & Número & Páginas & Autor \\
\hline 21-4-1838 & \multirow{19}{*}{$A T h Z$} & 80 & $355-356$ & \multirow{19}{*}{ Adami } \\
\hline $25-4-1838$ & & 83 & $366-367$ & \\
\hline $1-5-1838$ & & 87 & 383 & \\
\hline $5-5-1838$ & & 90 & $399-400$ & \\
\hline $8-5-1838$ & & 92 & $406-407$ & \\
\hline $10-5-1838$ & & 94 & $415-416$ & \\
\hline $16-5-1838$ & & 98 & 431 & \\
\hline $21-5-1838$ & & 101 & $447-448$ & \\
\hline $28-5-1838$ & & 106 & 470 & \\
\hline 21-11-1839 & & 234 & $1141-1142$ & \\
\hline $30-11-1839$ & & 240 & $1175-1176$ & \\
\hline $4-12-1839$ & & 243 & $1186-1187$ & \\
\hline $7-12-1839$ & & 245 & $1197-1198$ & \\
\hline $10-12-1839$ & & 247 & 1210 & \\
\hline $16-12-1839$ & & 251 & $1230-1231$ & \\
\hline $4-2-1840$ & & 30 & 122 & \\
\hline $11-2-1840$ & & 36 & 146 & \\
\hline $18-2-1840$ & & 42 & $170-171$ & \\
\hline $3-3-1846$ & & 53 & 211 & \\
\hline $7-3-1846$ & $W A M Z$ & 29 & 114 & A.S. \\
\hline $10-3-1846$ & \multirow{7}{*}{$A T h Z$} & 59 & 236 & Adami \\
\hline $11-3-1846$ & & 60 & 239 & Wiest \\
\hline 14-3-1846 & & 67 & 266 & \multirow{5}{*}{ Adami } \\
\hline $17-3-1846$ & & 65 & 260 & \\
\hline 19-3-1846 & & 67 & $274-275$ & \\
\hline $21-3-1846$ & & 69 & $274-275$ & \\
\hline 24 y $25-3-1846$ & & 71 y $72^{15}$ & $283-284$ & \\
\hline $26-3-1846$ & $W A M Z$ & 37 & 147 & Dr. K. \\
\hline $27-3-1846$ & \multirow{2}{*}{$A T h Z$} & 74 & 295 & Adami \\
\hline 28-3-1846 & & 75 & 299 & Anónimo \\
\hline $28-3-1846$ & $W A M Z$ & 38 & 150 & Anónimo \\
\hline $31-3-1846$ & \multirow{5}{*}{ AThZ } & 90 & 724 & Carl Walter \\
\hline $31-3-1846$ & & 77 & 307 & \multirow{4}{*}{ Adami } \\
\hline $2-4-1846$ & & 79 & 315 & \\
\hline 7-4-1846 & & 83 & 331 & \\
\hline $19-5-1846$ & & 119 & $474-475$ & \\
\hline
\end{tabular}


Todos los textos, a excepción del publicado el 11-3-1846, están reproducidos total o parcialmente en el libro de Dezső Legány Franz Liszt. Unbekannte Presse und Briefe aus Wien 1822-1886 ${ }^{16}$. Para este estudio, no obstante, se han consultado las fuentes originales, las cuales han revelado frases no recogidas por Legány y que contienen información relevante sobre los instrumentos usados por el compositor húngaro.

Como fuentes complementarias, se han utilizado un artículo del Allgemeine Musikalische Zeitung mit besonderer Rü̈ksicht auf den Österreichischen Kaiserstaat ${ }^{17}$ y el diccionario bibliográfico de la época Biographisches Lexikon des kaiserthums Oesterreich ${ }^{18}$.

\section{El piano en las décadas de 1830 y 1840}

Durante el s. XIX, el piano aún no era el instrumento estandarizado de hoy en día, sino que presentaba dos variantes de mecánica muy diferentes la una de la otra: la vienesa y la inglesa. La primera de ellas se caracterizaba por tener un tacto muy ligero y sensible a las órdenes de la mano del pianista, poco volumen sonoro, mucha claridad, una gran precisión en el apagado de las cuerdas y un calado exiguo. La segunda, en cambio, era menos directa en su respuesta, más pesada, menos transparente, con más calado y no muy limpia al apagar resonancias, pero permitía alcanzar una potencia sonora mayor y realizar con facilidad efectos de masa. A pesar de lo dicho, hay que tener en cuenta que hasta el piano inglés histórico más pesado y menos transparente es mucho más ligero y claro que cualquiera de los instrumentos actuales.

Antes de 1853, la encordadura de todo piano era recta, no cruzada, y no se empleaba aún el marco de hierro fundido, sino que, como mucho, algunos ejemplares tenían varias barras metálicas de refuerzo unidas a una plancha de ese mismo material, de modo que el elemento predominante en la estructura era la madera. Los macillos solían estar forrados con cuero, aunque poco a poco algunos pianos de mecánica inglesa iban incorporando el fieltro, revestimiento patentado por Pape en $1826^{19}$. Por otro lado, las teclas no eran tan anchas como las del piano moderno, lo que hacía posible abarcar extensiones grandes fácilmente, aunque el tamaño de la mano del pianista fuera reducido.

\footnotetext{
${ }^{15}$ Se trata de un número doble.

${ }^{16}$ Dezső Legány: Franz Liszt. Unbekannte Presse und Briefe aus Wien 1822-1886, Viena-Colonia-Graz, Böhlau Verlag, 1984.

${ }^{17}$ Allgemeine Musikalische Zeitung mit besonderer Rücksicht auf den Österreichischen Kaiserstaat, Viena, Steiner, 1817-1824.

${ }^{18}$ C. von Wurzbach: Biographisches Lexikon...

${ }^{19}$ François Lesure, Alain Roudier, Alain Moysan, Patricia Faivre, Anik Devriès, Paul Raspé: Sébastien Erard, 1752-1831 ou La rencontre avec le pianoforte: Luxeuil-les-Bains du 3 au 29 mai 1993, Luxeuil-Les-Bains, s.n, 1993, p.10.
} 
Otro rasgo de los pianos históricos que hay que tener en cuenta es que la estabilidad de su ajuste y afinación siempre fue problemática. Como ya se ha explicado con anterioridad, su principal elemento constructivo es la madera, material que reacciona enseguida a los cambios de humedad del ambiente: si baja el porcentaje, partes como la tabla armónica, el clavijero, los macillos y hasta las mismas paredes del mueble encogen; si sube, todos esos componentes se hinchan. En el primer caso, la afinación desciende y el estrechamiento de los laterales puede provocar que el fondo del clavijero llegue a abombarse, impidiendo el funcionamiento de la parte central del mecanismo. Los elementos de este último también pueden ir excesivamente sueltos, ocasionando irregularidades en el funcionamiento del escape y del atrape. En el segundo supuesto, la tensión de las cuerdas aumenta porque el puente asciende con la hinchazón de la tapa armónica, lo cual hace que se suba la afinación y que se rompan cuerdas con facilidad. Además, al crecer la madera de las partes del mecanismo, este empieza a moverse torpemente y algunas teclas pueden incluso quedarse atascadas. Los desajustes son, por otro lado, mucho más patentes en la mecánica vienesa que en la inglesa, y la razón para ello es que, al constar la primera de muchas menos palancas que la segunda, el mal funcionamiento de una de ellas afecta a un porcentaje bastante grande del proceso de producción del sonido. Como es bien sabido, todos estos inconvenientes se solucionaron con la aparición del piano moderno, que es aquel que aúna mecánica inglesa, encordadura cruzada y marco de hierro fundido en una sola pieza ${ }^{20}$. Por desgracia, los avances destinados a aumentar la fiabilidad del instrumento llevaron aparejada una pérdida de capacidad expresiva, de sensibilidad al toque y de transparencia.

\section{8: una inundación y un reencuentro}

Cuando Liszt, que a la sazón se encontraba en Venecia, leyó en los periódicos la noticia de las inundaciones que el 15 de marzo de 1838 habían asolado Pest y otras localidades cercanas ribereñas del Danubio, sintió en su interior una poderosa llamada a ayudar a sus compatriotas damnificados ${ }^{21}$. La manera que él tenía de contribuir era organizando conciertos benéficos y donando la recaudación de los mismos a la causa, de modo que, a los pocos días de tener conocimiento de la tragedia, ya estaba dando una serie de ocho actuaciones públicas en Viena, que tuvieron lugar entre el 18 de abril y el 25 de mayo de ese año. Hacía quince años

\footnotetext{
${ }^{20}$ Los primeros pianos modernos fueron fabricados por Chickering y Steinway en torno a 1860. No obstante, su penetración en el mercado fue paulatina y no lo dominó hasta algunas décadas más tarde, ya que hasta la Segunda Guerra Mundial se siguieron produciendo bastantes instrumentos que no presentaban alguno de los tres elementos constructivos mencionados.

${ }^{21}$ Alan Walker: Franz Liszt. The virtuoso years 1811-1847, Ithaca (NY), Cornell University Press, 1987, pp. 253-254.
} 
que el músico no visitaba la capital austríaca, en la que había realizado estudios con Czerny y Salieri y en la que había subido por última vez al escenario el 13 de abril de $1823^{22}$.

En su infancia y en los albores de la adolescencia, Liszt había tocado exclusivamente pianos de mecánica vienesa, que eran los que estaban disponibles en la zona de Europa en la que vivía. Los de las décadas de 1810-1820 tenían, por lo general, una extensión de seis octavas y solían estar dotados de un gran número de pedales, que accionaban registros muy variopintos: el de resonancia, que levantaba los apagadores; el moderador, que introducía una tira de material textil entre macillo y cuerda para dulcificar el sonido; el de desplazamiento, que funcionaba como el actual pedal izquierdo; el de fagot, que imitaba el sonido de dicho instrumento de viento al poner en contacto un pergamino con las cuerdas de la región media-grave; y el de música turca, que constaba de bombo, platillos y campanillas (percusión habitual en las bandas de músicos jenízaros).

Al instalarse en París a finales de 1823, el jovencísimo Liszt se encontró por primera vez con el piano de mecánica inglesa, cuyo representante más avanzado técnicamente era Erard, marca de la cual él se convirtió enseguida en artista patrocinado. Dicha colaboración le permitía disponer de pianos para su uso personal, a cambio de emplearlos luego en sus actuaciones ${ }^{23}$. Como cualquier otro pianista del momento, Liszt en esos años también tocaba con regularidad ejemplares de otros fabricantes, como Pleyel, Herz, Pape, Ditz, Petzold o Klepfer ${ }^{24}$.

Los conciertos de 1838 en Viena le trajeron a Liszt dos sorpresas: la primera fue el éxito de público sin precedentes con el que se encontró, tal y como se desprende de la correspondencia de esas semanas entre el compositor y su amante Marie d'Agoult ${ }^{25}$; la segunda consistió en su reencuentro con la mecánica vienesa, a través de los pianos del fabricante Conrad Graf. Los instrumentos de esos años habían aumentado de extensión de teclado, llegando a abarcar las seis octavas y media (de Do1 a Sol7), y habían perdido varios pedales (se mantenían el de resonancia, el de desplazamiento y, a veces, el moderador). Los macillos, aún revestidos de finas capas de cuero, se habían tornado ligeramente más grandes con objeto de poder golpear cuerdas que estaban más tensas y que tenían mayor grosor que en los pianos construidos con anterioridad. Dicho aumento de tensión estaba relacionado con la creciente necesidad de dotar de un mayor volumen sonoro a los instrumentos, de modo que pudieran resultar suficientes para poderse oír sin problemas en un teatro relativamente grande. La longitud habitual de los pianos de cola oscilaba entre $2 \mathrm{~m} 30 \mathrm{~cm}$ y $2 \mathrm{~m} 47 \mathrm{~cm}$, y el único refuerzo de metal que contenían era una pequeña barrita camuflada entre los macillos y

\footnotetext{
${ }^{22}$ Allgemeine Musikalische Zeitung..., n. ${ }^{\circ}$ 34, p. 270.

${ }^{23}$ A. Walker: Franz Liszt. The virtuoso years..., pp. 492-493.

${ }^{24}$ G. Keeling: "The Liszt Pianos...", p. 221.

${ }^{25}$ Serge Gut, Jacqueline Bellas (eds.): Correspondance Franz Liszt Marie d’Agoult, París, Fayard, 2001, pp. 309-335.
} 
alineada con estos bajo la encordadura. Su sonido era ligero, pero algo más cálido que el de principios de siglo. El tacto era muy agradable, con muy poca resistencia y un calado relativamente pequeño.

Como se puede ver en el cuadro 1, que se halla al final de esta sección y que recoge los detalles de las actuaciones públicas (fechas, pianos y repertorio), Liszt dio el primero de sus conciertos el 18 de abril ${ }^{26}$. Fue una ocasión un tanto especial, pues en ella se sirvió de tres pianos: un Erard (mecánica inglesa) y dos Graf (mecánica vienesa). Adami señala que saltaron algunas cuerdas, pero que de tal cosa, al haber sucedido "en una ejecución tan marcada y enérgica, no podía echárseles la culpa a los instrumentos" 27 . En cambio, del evento del 23 de abril, en el cual Liszt usó dos pianos de Graf, Adami escribe que "esta vez aguantaron tanto las cuerdas como la afinación, lo cual resultaba del todo sorprendente, teniendo en cuenta la temperatura que hacía en la sala del concierto" ${ }^{28}$. De las actuaciones del 29 de abril y de las del 2, 6, 8,14 y 18 de mayo, únicamente se sabe cuáles fueron los pianos que utilizó el compositor húngaro, pero no hay información alguna acerca de cómo respondieron. Es posible que el crítico no haya escrito nada al respecto porque no se hubiera producido ningún incidente destacable en el transcurso de la ejecución.

El concierto de despedida de la serie vienesa de 1838 se celebró el 25 de mayo. En su crítica, Adami declara que Liszt había tocado, tanto en esa actuación como en las previas, siempre en el mismo piano de Graf, aunque el Erard de Thalberg había estado a su disposición (es posible que este instrumento concreto fuera el que usó para el Konzertstück de Weber el 18 de abril y el 6 de mayo). Según el texto, los ejemplares del fabricante vienés "habían pasado una verdadera prueba de fuego" 29 bajo las manos del pianista húngaro. Esa afirmación de Adami hay que entenderla tanto en relación a la técnica pianística de Liszt como al repertorio que este llevaba en la gira, el cual presentaba unas dificultades y unos efectos nunca vistos hasta entonces. La escritura de esas obras, de tipo orquestal y muy colorístico, no se podía abordar con los tipos de toque habituales en el enfoque mecánico general de la época, que se limitaba a atacar de dedo y muy de cerca, manteniendo inmóviles el brazo y la mano. La realidad es que los desplazamientos rápidos sobre las teclas, las abigarradas texturas y la vigorosa acentuación que están presentes en la escritura lisztiana de los años de virtuoso son irrealizables, aun en los ligeros pianos del momento, sin una cierta participación del brazo, así que seguramente fueron estos gestos del maestro los que, por su mayor amplitud y energía que los acostumbrados en otros intérpretes, sorprendieron a Adami y le hicieron pensar que la integridad fisica de los instrumentos corría peligro. No obstante, no hay que pasar por alto que,

\footnotetext{
${ }^{26}$ Todos los datos de los conciertos están recogidos de las críticas de Adami, salvo que se indique lo contrario.

${ }^{27}$ AThZ, 21-4-1838, p. 356. Todos los artículos de prensa que han servido como fuentes están en alemán. Las traducciones al español son de la autora.

${ }^{28}$ AThz, 25-4-1838, p. 367.

${ }^{29}$ AThZ, 28-5-1838, p. 470 .
} 
si Liszt escogió repetidamente el Graf frente al Erard, es porque él mismo confiaba en la capacidad que tenía el instrumento vienés para salir airoso de las situaciones comprometidas.

El siguiente cuadro muestra los pianos, las mecánicas de estos y el repertorio de los conciertos vieneses de Liszt en 1838:

Cuadro 1. Conciertos de 1838

\begin{tabular}{|c|c|c|c|}
\hline Fecha & Pianos & Mecánica & Repertorio \\
\hline \multirow[t]{2}{*}{$18-4$} & Erard & Inglesa & Weber: Konzertstück. \\
\hline & Graf (dos) & Vienesa & $\begin{array}{l}\text { Acompañamiento de Lied (Beethoven: Adélaïde). } \\
\text { Liszt: Réminiscences des Puritains R. } 129^{30} \text {, Valse de bravour R. } 32 a \text { y } \\
\text { Grande étude en Sol mineur R. } 2 a / 6^{31} \text {. }\end{array}$ \\
\hline $23-4$ & Graf (dos) & Vienesa & $\begin{array}{l}\text { Hummel: Septeto. } \\
\text { Schubert-Liszt: Ständchen R. } 243 / 9 \text { y Lob der Thränen R. } 242 . \\
\text { Pacini-Liszt: Divertissement sur la cavatine de Pacini 'I tuoi frequenti palpiti' } \\
\text { R. } 230 . \\
\text { Acompañamiento de ópera (Cavatina 'I tuoi frequenti palpiti). }\end{array}$ \\
\hline $29-4$ & Graf & Vienesa & $\begin{array}{l}\text { Czerny: Sonata en La bemol mayor. } \\
\text { Acompañamientos de Lieder (Schubert: Liebesbotschaft y Erlkönig). } \\
\text { Moscheles y Chopin: cuatro estudios. } \\
\text { Liszt: Grande fantaisie sur des motifs des Soirées musicales R. } 234 .\end{array}$ \\
\hline $2-5$ & $\mathrm{Graf}^{32}$ & Vienesa & $\begin{array}{l}\text { Beethoven: Sonata en La bemol mayor }{ }^{33} \text {. } \\
\text { Acompañamientos de Lieder (Schubert: Ständchen y Der Hirt auf dem } \\
\text { Felsen). } \\
\text { Meyerbeer-Liszt: Grande fantaisie sur des thèmes de l'opéra Les Huguenots } \\
\text { (Réminiscences des Huguenots) R. } 221 \text {. } \\
\text { Liszt: noveno de los Grandes Études }{ }^{34} \text { y Grande galop chromatique R. } 41 .\end{array}$ \\
\hline $6-5$ & Erard & Inglesa & Weber: Konzertstück. \\
\hline $8-5$ & Graf & Vienesa & $\begin{array}{l}\text { Liszt: Réminiscences de la Juive R. } 170 . \\
\text { Moscheles y Chopin: cuatro estudios. } \\
\text { Acompañamiento de Lied (Schubert: Der Wanderer). } \\
\text { Hexaméron R. } 131^{35} \text {. }\end{array}$ \\
\hline
\end{tabular}

${ }^{30}$ La identificación de las obras de Liszt se hace colocando el número del catálogo de Raabe tras el título. Este último se mantiene en su forma e idioma originales, salvo en el caso de denominaciones genéricas (sonata, romanza, mazurka, etc.).

${ }^{31}$ Se trata de la versión de 1838 de lo que será el Estudio de ejecución trascendental n. * 6, "Visión".

${ }^{32}$ En las críticas tanto de este concierto como del celebrado el 14 de mayo, Adami no menciona el piano que se empleó. Hay que avanzar hasta la publicada con ocasión de la despedida para leer que "Liszt había tocado en todos sus conciertos en el mismo excelente instrumento del Sr. Graf' (AThZ, 28-5-1838, p. 470).

${ }^{33}$ Las sonatas de Beethoven que están escritas en esta tonalidad son la op. 26 y la op. 110.

${ }^{34}$ Se trata de la versión de 1838 del Ricordanza (R. 2a/9).

${ }^{35}$ Esta es una obra de autoría compartida entre seis pianistas: Liszt, Czerny, Herz, Pixis, Chopin y Thalberg. Cada uno de ellos hizo una de las variaciones sobre el tema (la Marcha de Des Puritains), pero Liszt se encargó, además, de componer la introducción, el arreglo del tema, la variación final y las transiciones. 


\begin{tabular}{|c|c|c|c|}
\hline Fecha & Pianos & Mecánica & Repertorio \\
\hline $14-5$ & Graf & Vienesa & $\begin{array}{l}\text { Beethoven: Sonata op. } 27 n^{\circ} 2 . \\
\text { Acompañamientos de Lieder (Schubert: Das Fischermädchen, Der } \\
\text { Kreuzzug y Die Forelle; dos Lieder de Randhartinger). } \\
\text { Kessler: Estudio en La bemol mayor y el Estudio de octavas. } \\
\text { Haendel: Fuga en mi menor. } \\
\text { Scarlatti: Fuga del gato. } \\
\text { Hexaméron R. } 131 .\end{array}$ \\
\hline $18-5$ & Graf & Vienesa & $\begin{array}{l}\text { Liszt: Fantasía romántica sobre una canción suiza R. } 9 . \\
\text { Weber: Aufförderung zum Tanz. } \\
\text { Acompañamientos de Lieder (Schubert: Auf dem Strom y Die Post). } \\
\text { Rossini-Liszt: Soirées musicales de Rossini R. } 236 \text { (I marinari y La tarantella). } \\
\text { Improvisación libre. } \\
\text { Schubert-Liszt: Erlkönig. }\end{array}$ \\
\hline $25-5$ & Graf & Vienesa & $\begin{array}{l}\text { Rossini-Liszt: Ouverture de l'opéra Guillaume Tell R. } 237 . \\
\text { Acompañamientos de dos Lieder (Vesque von Puttlingen). } \\
\text { García-Liszt: Rondeau fantastique sur un thème espagnol (El contraban- } \\
\text { dista) R. } 88 \text {. } \\
\text { Berlioz-Liszt: dos fragmentos de la Sinfonie Fantastique de Berlioz } \\
\text { (R. } 134 \text { y 136). } \\
\text { Schubert-Liszt: Erlkönig R. } 243 / 1 \text {, Die Post R. } 243 / 4 \text { y Sei mir } \\
\text { gegrüß R. } 246 / 4) \text {. } \\
\text { Liszt: Valse de bravour R. } 32 a \text {. }\end{array}$ \\
\hline
\end{tabular}

\section{9: nuevos conciertos}

En otoño de 1839, Liszt viajó nuevamente a Viena para una serie de siete actuaciones públicas. Las críticas de Adami especifican que el piano utilizado en los tres primeros conciertos ( 19 y 27 de noviembre y 2 de diciembre) fue un Graf, pero no dicen nada ni de cómo se comportó ni de cuál fue el instrumento empleado en los otros cuatro (días 5 -dos eventos-, 8 y 14 de diciembre).

Este cuadro muestra la visión general de esta serie, en la que se aprecia la importancia creciente que tuvieron las fantasías sobre temas operísticos, que son obras con una escritura muy exigente para los instrumentos:

Cuadro 2. Conciertos de 1839

\begin{tabular}{|l|l|l|l|}
\hline Fecha & Pianos & Mecánica & Repertorio \\
\hline $19-11$ & Graf & Vienesa & $\begin{array}{l}\text { Beethoven-Liszt: tres últimos movimientos de la Sinfonía Pastoral } \\
(\text { R. 128//6). } \\
\text { Acompañamiento de Lied (Curci: Il platano). } \\
\text { Hexaméron R. 131. } \\
\text { Schubert-Liszt: Ave Maria R. 243/12, Ständchen R. 243/9. } \\
\text { Liszt: noveno de los Grandes Études }{ }^{36} .\end{array}$ \\
\hline $27-11$ & Graf & Vienesa & $\begin{array}{l}\text { Bellini-Liszt: Réminiscences des Puritains R. } 129 . \\
\text { Donizetti-Liszt: Réminiscences de Lucia R. 151. } \\
\text { Schubert-Liszt: Die Stadt R. 245/1, Das Fischermädchen R. 245/2, } \\
\text { Aufenthalt R. 245/3. }\end{array}$ \\
\hline
\end{tabular}

${ }^{36}$ La versión de 1838 del Mazeppa (R. 2a/9). 


\begin{tabular}{|c|c|c|c|}
\hline Fecha & Pianos & Mecánica & Repertorio \\
\hline $2-12$ & Graf & Vienesa & $\begin{array}{l}\text { Beethoven: Sonata op. } 57 . \\
\text { Acompañamientos de Lieder (Randhartinger: Das Erkennen; Schubert: } \\
\text { Die Forelle). } \\
\text { Donizetti-Liszt: Réminiscences de Lucia R. } 151 . \\
\text { Schubert-Liszt: Der Atlas R. } 245 / 11 \text { y Die Taubenpost R. 245/13). } \\
\text { Lickl: Am Kalvarien-Berg. } \\
\text { Liszt: Grande galop chromatique R. } 41 .\end{array}$ \\
\hline $5-12$ a & Sin determinar & Sin determinar & $\begin{array}{l}\text { Liszt: versión temprana de su Après un lecture du Dante (probable- } \\
\text { mente R. } 10 b \text { ), Valse de bravour R. } 32 a \text {. } \\
\text { Bellini-Liszt: Fantaisie sur des motifs favoris de l'opéra La sonnambula } \\
\text { R. } 132 . \\
\text { Acompañamiento de Lied (Beethoven: Adélaïde). } \\
\text { Chopin: mazurkas. }\end{array}$ \\
\hline $5-12 b$ & Sin determinar & Sin determinar & Beethoven: Concierto $n .^{\circ} 3$ para piano y orquesta. \\
\hline $8-12$ & Sin determinar & Sin determinar & $\begin{array}{l}\text { Beethoven: Sonata op. } 31 n .^{\circ} 2 . \\
\text { Kreutzer: Pastoral "Am Waldstädtersee". } \\
\text { Liszt: séptimo de los Grandes Études }{ }^{37} \text {, Tarantella R. } 132 . \\
\text { Rossini-Liszt: L'Orgia R. } 236 / 11 . \\
\text { Schubert-Liszt: Erlkönig R. } 10 a / 3) .\end{array}$ \\
\hline $14-12$ & Sin determinar & Sin determinar & $\begin{array}{l}\text { Beethoven: Trío en Si bemol mayor. } \\
\text { Acompañamientos de Lieder (Kreutzer; Beethoven: An die ferne Geliebte). } \\
\text { Schubert-Liszt: Mélodies hongroises R. } 250 . \\
\text { Bellini-Liszt: Fantaisie sur des motifs favoris de l'opéra La sonnambula R. } 132 .\end{array}$ \\
\hline
\end{tabular}

\section{0: retorno a la capital austríaca tras una breve ausencia}

Menos de dos meses después de su último concierto allí, Liszt emprendió una nueva serie de actuaciones enViena, que tuvieron lugar los días 2, 6, 9 y 14 de febrero. Adami recoge en la crítica al último de esos eventos que el piano utilizado siempre había sido un Graf, y lo hace elogiándolo con las siguientes palabras:

El magistral instrumento de Graf, que se había hecho famoso durante todos los conciertos previos de Liszt, cumplió también esta vez con su obligación y resultó ser completamente suficiente, incluso en esta sala de gran tamaño, tanto en los pasajes potentes y enérgicos, en los que entraba en competición con la orquesta, como en los que eran piano ${ }^{38}$.

Como se aprecia en el texto, la preocupación por la cantidad de sonido que fueran capaces de producir los pianos era grande. No solo tenían que conseguir llenar salas de cierto tamaño, sino que debían enfrentarse a unas orquestas cuyo número de integrantes iba en aumento y que tocaban con unos instrumentos que, gracias a los avances tecnológicos que también se estaban produciendo en ellos, poseían un techo sonoro cada vez más alto.

\footnotetext{
${ }^{37}$ Se trata de la versión de 1838 del Eroica (R. 2a/7).

${ }^{38}$ AThZ, 18-2-1840, p. 171.
} 
El piano que empleó Liszt en esos conciertos aparece en un famoso óleo de Danhauser, encargado por el fabricante al pintor en ese año ${ }^{39}$. Presenta un diseño especial con volutas en las patas, lo cual permite identificarlo con el n. ${ }^{\circ} 2787$, que se halla preservado en el Kunsthistorisches Museum deViena ${ }^{40}$.

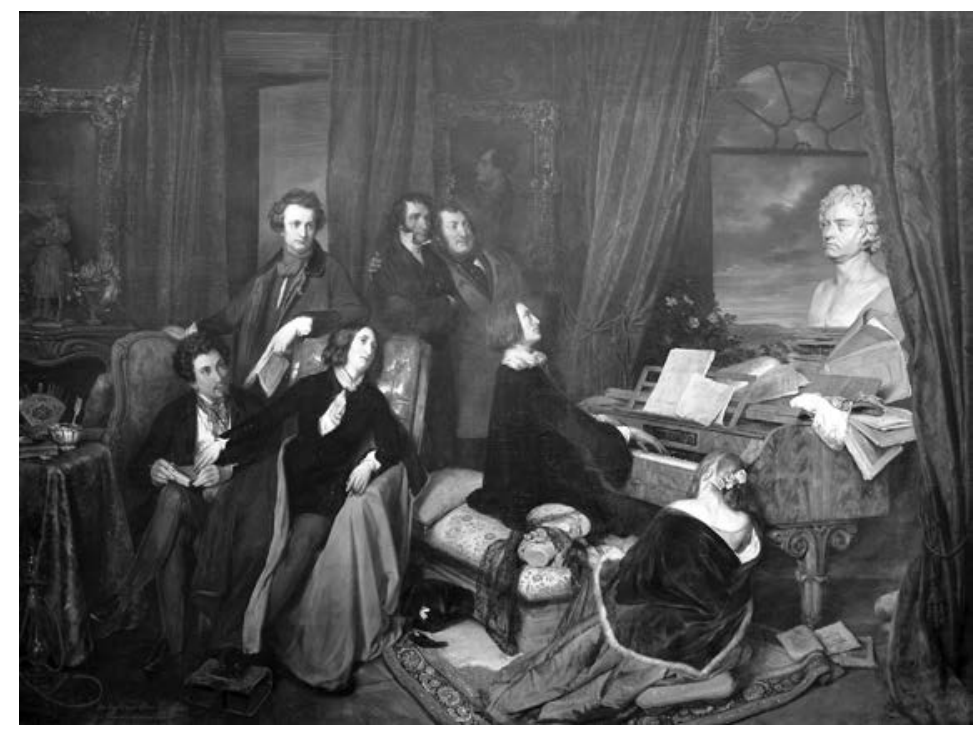

Liszt al piano (Danhauser)

El cuadro general de los conciertos de 1840 se presenta como sigue:

Cuadro 3. Conciertos de 1840

\begin{tabular}{|c|c|c|c|}
\hline Fecha & Pianos & Mecánica & Repertorio \\
\hline $2-12$ & Graf & Vienesa & $\begin{array}{l}\text { Mercadante-Liszt: Fantasía sobre "Il giuramento" R. } 294 . \\
\text { Schubert-Liszt: Erlkönig R. } 243 / 4 \text {, dos números del Winterreise y } \\
\text { Mélodies hongroises R. } 250 . \\
\text { Desconocido: Marcha. }\end{array}$ \\
\hline $6-12$ & Graf & Vienesa & $\begin{array}{l}\text { Schubert-Liszt: Erlkönig R. 243/4, Ständchen R. } 243 / 9 \text { y Mélodies } \\
\text { hongroises R. } 250 . \\
\text { Bellini-Liszt: Fantaisie sur des motifs favoris de l'opéra La sonnambula } \\
\text { R. } 132 \text {. } \\
\text { Auber-Liszt: Grande fantaisie sur la tyrolienne de l'opéra la Fiancée R. } \\
\text { 116. }\end{array}$ \\
\hline $9-12$ & Graf & Vienesa & $\begin{array}{l}\text { Meyerbeer-Liszt: Grande fantaisie sur des thèmes de l'opéra Les Hugue- } \\
\text { nots (Réminiscences des Huguenots) R. } 221 . \\
\text { Mercadante-Liszt: Fantasía sobre "Il giuramento" R. } 294 . \\
\text { Weber: Aufförderung zum Tanz. } \\
\text { Donizetti-Liszt: Réminiscences de Lucia R. } 151 . \\
\text { Liszt: dos de los Études d'éxecution trascendante d'après Paganini R. 3a. } \\
\text { Chopin: estudio. }\end{array}$ \\
\hline
\end{tabular}

${ }^{39}$ El cuadro se encuentra en la actualidad en el Musikinstrumenten-Museum de Berlín.

${ }^{40}$ Martha Novak Clinkscale: Makers of the piano 1820-1860, Oxford University Press, 1993, p. 158. 


\begin{tabular}{|l|l|l|l|}
\hline Fecha & Pianos & Mecánica & Repertorio \\
\hline $14-12$ & Graf & Vienesa & Weber: Konzertstück. \\
& & Beethoven: Fantasía coral. \\
& & $\begin{array}{l}\text { Hummel: Septeto. } \\
\text { Pacini-Liszt: Divertissement sur la cavatine de Pacini 'I tuoi frequenti } \\
\end{array}$ & \\
& & palpiti' R. 230. \\
& & Acompañamiento de Lied (Hoven: In die Ferne). \\
\hline
\end{tabular}

\section{6: entran en escena Streicher y Bösendorfer}

Seis años después de su última serie de conciertos, Liszt volvió a Viena a ofrecer varias actuaciones más. Algunas cosas habían cambiado en el mundo de la construcción de pianos: Conrad Graf había vendido en 1840 su taller y nuevos fabricantes empezaban a cubrir el hueco resultante (entre ellos, Streicher y Bösendorfer). Por otra parte, los instrumentos vieneses veían cómo la mayor potencia sonora de los ingleses les iba comiendo terreno, así que empezaron a sufrir modificaciones, tales como la incorporación de barras metálicas de refuerzo (algo que la competencia llevaba décadas utilizando) y el aumento de la tensión de las cuerdas y, por consiguiente, del tamaño de los macillos. Esto último, de hecho, supuso el inicio del lento declive del piano vienés, ya que su principal ventaja había sido hasta ahora la ligereza y la precisión, pero unos macillos más grandes y pesados convertían al mecanismo en un sistema más torpe. Además, marcas como Streicher o Bösendorfer empezaban a experimentar con las mecánicas inglesa y semi-inglesa (una variante muy simple de la inglesa, sin rodillo de repetición, que se extendió bastante en Centroeuropa en la segunda mitad del s. XIX).

El primero de los conciertos de esta tanda lo dio Liszt el 1 de marzo. Según Adami, tuvo dos pianos de Streicher en el escenario, pero solo empleó uno de ellos, que estaba provisto de mecánica inglesa. Dice de él:

El único instrumento del que se sirvió en este concierto, un piano de cola extraordinariamente elaborado y sonoro de mecánica inglesa, demostró ser realmente excelente, mantuvo muy bien la afinación tras una sucesión de tantas piezas y no se le rompió más que una cuerda en el bajo, algo que no impidió continuar tocando ${ }^{41}$.

Liszt ya había usado en alguna ocasión pianos de este fabricante. De hecho, en su carta del 1 de febrero a Pierre Erard escribe, hablando de sus dificultades con un Pleyel:"al día siguiente [...], le rogué al príncipe Hercolani [sic] que me prestara un piano de Streicher de Viena, que me vino infinitamente mejor"42. Keeling afirma, además, que:

${ }^{41}$ AThz, 3-3-1846, p. 211

${ }^{42}$ Franz Liszt, Michael Saffle, Michael Short (eds.): Liszt Letters in the Library of Congress, Hillsdale (NY), Pendragon Press, 2003, p. 7. 
Liszt seguramente había estado expuesto a los instrumentos de Streicher ya en tiempos de sus estudios tempranos enViena, ya que su profesor Carl Czerny escribió en 1823 en el cuaderno de conversaciones de Beethoven que 'solo me gustaban sus instrumentos [de Streicher] ${ }^{\text {43 }}$.

En realidad, lo más probable es que esos instrumentos de esa fábrica no fueran obra del propio Johann Baptist Streicher, que fue quien construyó los que Liszt manejó de 1838 en adelante, ya que hasta 1823 su madre Nanette no lo tomó como compañero en la empresa familiar ${ }^{44}$, y justo a finales de ese año fue cuando el joven pianista húngaro dejóViena para establecerse en París.

Liszt dio su tercer concierto el 8 de marzo. En esa ocasión, usó dos Streicher, de los cuales el primero dio algún que otro problema:

El excesivo calor reinante en la sala puede haber sido también el culpable de que hayan saltado por los aires algunas cuerdas en el piano de Streicher, a pesar de que Liszt no hubiera forzado el instrumento en absoluto. Este mismo fue sustituido por un segundo piano de bello sonido, que aguantó eficientemente hasta el final ${ }^{45}$.

La alusión a la alta temperatura como causante de problemas mecánicos en los pianos no es nueva en las críticas de Adami.Ya en la del concierto del 23 de abril de 1838 había manifestado su sorpresa por lo bien que había aguantado el instrumento, a pesar del calor que hacía en la sala ${ }^{46}$. La realidad es que no son en sí las oscilaciones de temperatura las que desbaratan la afinación y el ajuste, sino las de la humedad ambiental. Lo que ocurre es que esta última fluctúa con la primera, a no ser que se emplee un medio artificial para regularla (un humidificador o un deshumidificador, según la necesidad, artilugio que, evidentemente, en tiempos de Liszt no existía). Además, en muchos casos, los pianos procedían de tiendas o de casas particulares, y estos espacios tenían, generalmente, un aire mucho más seco que las salas abarrotadas de público. Cuando, tras el transporte -efectuado muchas veces en condiciones precarias-, el instrumento llegaba al escenario, comenzaba a sufrir los efectos del vapor de agua que exhalaba el público con su respiración. Como es bien sabido, la madera se hincha con la humedad, y este es precisamente el material del que está hecha la tapa armónica. $\mathrm{Al}$ aumentar de tamaño dicha parte del instrumento, el puente sube y estira las cuerdas más allá de su tensión deseable, lo cual acaba por romperlas, previa desafinación de las mismas. En los instrumentos históricos saltan a veces solas, sin que nadie esté tocando en ese momento. La razón de esto es que tienen menos calibre y, en consecuencia, son más frágiles que las del piano actual.

\footnotetext{
${ }^{43}$ Los corchetes están en el original. G. Keeling: "Liszt and J. B. Streicher...", p. 36.

${ }^{44}$ Martha Novak Clinkscale: Makers of the piano 1700-1820, Oxford, Oxford University Press, 1993, p. 290.

${ }^{45}$ AThz, 10-3-1838, p. 236.

${ }^{46}$ AThZ, 25-4-1838, p. 367.
} 
El 11 de marzo, Liszt ofreció su cuarto concierto de esta serie. El piano fue nuevamente un Streicher, del cual dice Adami que "esta vez no sufrió ningún percance" ${ }^{47}$. Precisamente ese mismo día, Franz Wiest publicaba en el $A T h Z$ una columna de carácter humorístico sobre Liszt, en la que se pueden leer algunas frases relativas a los instrumentos que el compositor había utilizado en sus actuaciones:

Liszt es muy amable como persona y como artista. Nadie puede permanecer mucho tiempo tenso en su presencia, y en ningún caso las cuerdas del piano, ique le saltan alegremente por todas partes!

Liszt se me representa como un augurio romano, que adivina mediante las entrañas de aves de corral sacrificadas. La fábrica de aves de Streicher ya se ha desangrado ${ }^{48}$.

Liszt ha tenido dos artistas trágicos como colaboradores en su tercer concierto: los dos sirvientes de la casa Streicher, que tuvieron que llevarse el piano caído en combate ${ }^{49}$.

Las palabras de Wiest dejan ver que los incidentes en escena eran frecuentes y que, además, eran algo que llamaba lo suficientemente la atención como para merecer un espacio en una columna satírica.

El 15 de marzo, Liszt participó en dos conciertos. El primero de ellos supuso para Liszt el encuentro con pianos de un nuevo fabricante: Bösendorfer. Adami escribió en su crítica: "los dos instrumentos de Bösendorfer demostraron ser excelentes. Tocados por manos tan magistrales como las de Bocklet y Liszt, mostraron una vez más su sonido bello y poderoso" ${ }^{50}$. Del segundo concierto de ese día no se conoce más que el repertorio, ya que el texto del $A T h Z$ no dice nada del piano ${ }^{51}$.

La siguiente actuación pública de Liszt tuvo lugar el 17 de marzo. En ella, el maestro se sirvió de un piano de Streicher. En su crítica,Adami incluye un curioso texto en el que expresa su deseo de comparar los instrumentos de ese fabricante con los de Bösendorfer, incitando a una especie de duelo entre ambos:

Las cuerdas [del Streicher] aguantaron excelentemente, pero la afinación fue perdiéndose poco a poco, lo cual no resulta en absoluto sorprendente si se tiene en cuenta el tremendo calor que se había apoderado de la sala. La excelencia de estos instrumentos viene reconociéndose desde hace tiempo y, sin querer en lo más mínimo ofender a su fabricante, sería deseable que Liszt utilizara un piano de Bösendorfer en uno de sus próximos conciertos, para ver cómo pasa la prueba

${ }^{47}$ AThZ, 14-3-1846, p. 251

${ }^{48}$ Este párrafo es la traducción literal de un texto que emplea juegos de palabras: en alemán, "piano de cola" se dice "Flügel", que significa también "ala", y "ave de corral" es "Geflügel".

${ }^{49}$ AThZ, 11-3-1846, p. 239

${ }^{50}$ AThZ, 17-3-1846, p. 260

${ }^{51}$ AThZ, 18-3-1846, p. 264. 
de fuego. El público expresó varias veces ese deseo, y sería deseable e inspirador que a un empresario tan activo y respetable se le permitiera llevar sus pianos al campo de batalla en una ocasión así. Queremos ver cómo resisten ${ }^{52}$.

El 21 de marzo, Liszt participó en un evento benéfico. En esta ocasión, Adami no identifica el piano que se usó. Pero del siguiente concierto, que tuvo lugar el 22 de marzo, sí se sabe que Liszt tuvo dos Streicher en la sala, y Adami escribe que "el segundo de ellos destacó por su bello sonido y buena afinación" 53 . De este evento se conserva también la crítica aparecida en el $W A M Z$ y firmada por un tal "Dr. K.", pero en ella no se menciona a los instrumentos empleados ${ }^{54}$.

El 25 de marzo, la víspera del aniversario del fallecimiento de Beethoven, varios músicos rindieron homenaje al compositor de Bonn tocando juntos en concierto. Entre ellos estaba Liszt, que colaboró tocando con el violinista Ernst y con su antiguo profesor Czerny. Esta vez no fue Adami quien proporcionó los detalles acerca de los instrumentos empleados, sino Carl Walter, del Wiener Zeitung, quien escribió: "ambos fortepianos, de la fábrica del Sr. Carl Stein, aunque sufrieron un uso bastante exigente, mantuvieron todo el tiempo su bella y pura afinación" 55 . El constructor mencionado era miembro de la saga Stein-Streicher, nieto de Johann Andreas Stein, sobrino de Nanette Streicher y primo carnal de Johann Baptist Streicher ${ }^{56}$.

El 26 de marzo, Liszt tomó parte en dos eventos. La crítica del primero de ellos, del $W A M Z$ y anónima, no contiene mención alguna al piano utilizado. El segundo evento de ese día fue un concierto de Gustav Hölzel, en el que Liszt tuvo una pequeña intervención. La crítica, anónima y aparecida en el $A T h Z$, tampoco identifica el piano. En cambio, se sabe que al día siguiente el maestro tocó en un Erard, que Adami describe como "un instrumento de sonido claro y potente y de excelente mecánica" 57 . El 28 de marzo, el infatigable Liszt volvió a subirse al escenario. Dice Adami:

Esta vez se sirvió del conocido instrumento Bösendorfer de la exposición ${ }^{58}$, con mecánica de Erard, cuya excelencia mostró de sobra en esa calurosa velada. No solo mantuvo un sonido bello y pleno bajo tales manos maestras, sino que tanto

\footnotetext{
${ }^{52}$ AThz, 19-3-1846, p. 266.

${ }^{53}$ AThz, 24 y 25-3-1846, p. 284.

${ }^{54}$ WAMZ, 26-3-1846, p. 147.

${ }^{55} \mathrm{WZ}, 31-3-1846$, p. 724.

${ }^{56}$ Johann Andreas Stein fue el constructor preferido de Mozart. Su hija Nanette hizo también excelentes pianos.

${ }^{57}$ AthZ, 31-3-1846, p. 307.

${ }^{58}$ Adami se refiere aquí a la Exposición industrial de Viena de julio de 1845, en la que Bösendorfer había ganado la medalla de oro (WAMZ, 15-7-1845, p. 333).
} 
las cuerdas como la afinación resistieron hasta el final, lo cual no fue poco sorprendente, teniendo en cuenta las tres piezas de concierto que se habían hecho y la energía con que Liszt había manejado el piano ${ }^{59}$.

Liszt tuvo también una intervención muy breve en el concierto en honor a Beethoven del 29 de marzo. En esta ocasión, Adami no recoge nada acerca del piano, pero sí lo hace sobre el ejemplar del evento del 31 de marzo, escribiendo que "Liszt se sirvió en este concierto del mismo piano Bösendorfer que ya había tocado con anterioridad, el cual aguantó firme como el hierro de principio a fin"60.

Unos días después, el 4 de abril, Liszt volvió a usar "un instrumento de Streicher de excelente calidad que resistió perfectamente y cuya afinación no debió ser corregida ni una sola vez, a pesar de tantas piezas de concierto como se tocaron"61. El comentario de Adami es interesante porque muestra que en aquella época era habitual tener que repasar la afinación en medio de las actuaciones públicas.

Tras unos días de ausencia, a causa de una gira por Praga y Pest, Liszt dio el último concierto de sus años de virtuoso en Viena. El evento tuvo lugar el 17 de abril y en él se emplearon dos pianos distintos: un Erard y un Streicher. De ellos, dice Adami:

Ambos pianos, un Erard y un segundo del Sr. Streicher, se portaron impecablemente y resistieron perfectamente. Especialmente este último, un bonito y elegante instrumento en el que Liszt ejecutó todas sus piezas de bravura, se distinguió como el más ventajoso por su sonido pleno y potente ${ }^{62}$.

Las tres obras que iban con orquesta las tocó Liszt en el Erard, mientras que las piezas de bravura le correspondieron al Streicher. Las palabras del crítico no aclaran en qué piano se hicieron los acompañamientos vocales.

El cuadro general de esta serie de conciertos queda como sigue:

\footnotetext{
${ }^{59}$ Athz, 31-3-1846, p. 307.

${ }^{60}$ AThZ, 2-4-1846, p. 315.

${ }^{61}$ AThz, 7-4-1846, p. 331

${ }^{62}$ AThZ, 19-5-1846, p. 475.
} 
Cuadro 4. Conciertos de 1846

\begin{tabular}{|c|c|c|c|}
\hline Fecha & Pianos & Mecánica & Repertorio \\
\hline $1-3$ & $\begin{array}{l}\text { Streicher (dos, } \\
\text { pero solo usó } \\
\text { uno) }\end{array}$ & Inglesa & $\begin{array}{l}\text { Mozart-Liszt: Réminiscences de "Don Juan” R. } 228 . \\
\text { Schubert-Liszt: Erlkönig R. } 243 / 4 \text {, Ave Maria R. } 243 / 12 \text { y Mélodies } \\
\text { hongroises R. } 250 . \\
\text { Beethoven: Sonata op. } 27 \text { n. } 2 \text {. } \\
\text { Donizetti-Liszt: Marche funèbre de Dom Sébastien R. } 156 .\end{array}$ \\
\hline $7-3$ & Sin determinar & Sin determinar & $\begin{array}{l}\text { Rossini-Liszt: Ouverture de l'opéra Guillaume Tell R. } 237 . \\
\text { Liszt: las primeras versiones de Au lac de Wallenstadt R. } 10 a / 2 \text { y de } \\
\text { Au bord d'une source R. } 10 a / 4 \text { y Aires nacionales húngaros }{ }^{63} \text {. } \\
\text { Schubert: Fantasía Wanderer. } \\
\text { Chopin: dos estudios. } \\
\text { Bellini-Liszt: Réminiscences de Norma R. } 133 \text {. }\end{array}$ \\
\hline $8-3$ & Streicher (dos) & Vienesa $(?)^{64}$ & $\begin{array}{l}\text { Beethoven: Sonata op. } 57 . \\
\text { Bach: preludio y fuga. } \\
\text { Chopin: estudio. } \\
\text { Meyerbeer-Liszt: Réminiscences de Robert le diable R. } 222 . \\
\text { Liszt: uno de los Sonetti del Petrarca }{ }^{65} \text {. } \\
\text { Schubert-Liszt: Mélodies hongroises R. } 250 . \\
\text { Hexaméron R. } 131 .\end{array}$ \\
\hline $11-3$ & Streicher & Vienesa (?) & $\begin{array}{l}\text { Donizetti-Liszt: Réminiscences de Lucia R. } 151 . \\
\text { Bellini-Liszt: Fantaisie sur des motifs favoris de l'opéra La sonnambula R. 132). } \\
\text { Mendelssohn: Scherzo en Fa\# menor. } \\
\text { Schubert: Divertissement à la hongroise. } \\
\text { Chopin: dos mazurkas. } \\
\text { Czerny: Fantasía op. } 27 .\end{array}$ \\
\hline $15-3 \mathrm{a}$ & Bösendorfer & Vienesa & $\begin{array}{l}\text { Hummel: Sonata en La bemol mayor para piano a cuatro manos. } \\
\text { Acompanamientos de dos Lieder (Hovens). }\end{array}$ \\
\hline $15-3 b$ & Sin determinar & Sin determinar & $\begin{array}{l}\text { Bellini-Liszt: Fantaisie sur des motifs favoris de l'opéra La sonnambula R. } 132 . \\
\text { Donizetti-Liszt: Réminiscences de Lucia R. } 151 . \\
\text { Schubert-Liszt: Aires nacionales húngaros }{ }^{66} .\end{array}$ \\
\hline $17-3$ & Streicher & Vienesa (?) & $\begin{array}{l}\text { Beethoven: Sonata en La bemol mayoo }{ }^{67} \text {. Chopin: mazurka } \\
\text { Meyerbeer-Liszt: Grande fantaisie sur des thèmes de l'opéra Les Huguenots } \\
\text { (Réminiscences des Huguenots) R. } 221 . \\
\text { Berlioz-Liszt: Marche des pèlerins de la Sinfonie Harold en Italie R. } 139 . \\
\text { Rossini-Liszt: Tarantella (probablemente, la R. 236). } \\
\text { Bellini-Liszt: Réminiscences des Puritains R. } 129 . \\
\text { Schubert-Liszt: Die Taubenpost R. } 245 / 13 . \\
\text { Liszt: Grande galop chromatique R. } 41 .\end{array}$ \\
\hline $21-3$ & Sin determinar & Sin determinar & $\begin{array}{l}\text { Acompañamientos de Lied (Schubert: Erlkönig) y de ópera (Haendel: Acis } \\
\text { and Galatea). } \\
\text { Improvisación sobre los temas de las dos obras anteriores. }\end{array}$ \\
\hline $22-3$ & Streicher (dos) & Vienesa (?) & $\begin{array}{l}\text { Kullak: Paraphrase du quatrième acte de l'Opéra Dom Sébastien. } \\
\text { Schubert-Liszt: Zügenglöcklein (R. 248/3) y Die Forelle (R. 248/6). } \\
\text { Weber: Aufforderung zum Tanz. } \\
\text { Beethoven: Sonata op. } 101 . \\
\text { Donizetti-Liszt: Fantasie sur des motifs favoris de Lucrezia Borgia R. } 154 .\end{array}$ \\
\hline $25-3$ & Carl Stein (dos) & Vienesa & $\begin{array}{l}\text { Beethoven: Sonata op. } 106 \text {, Sonata "Kreutzer", transcripción para dos pianos } \\
\text { del primer movimiento de la 7. Sinfonía. }\end{array}$ \\
\hline
\end{tabular}

${ }^{63}$ Legány apunta la posibilidad de que se trate de la primera de las piezas identificadas con el número de catálogo R. 105d, tituladas Ungarische National-Melodien (D. Legány: Franz Liszt. Unbekannte..., p. 89).

${ }^{64}$ Los textos no especifican la mecánica que tenían los pianos de Streicher, pero se supone que, de no decirse lo contrario, serían vieneses.

${ }^{65}$ En su primera versión pianística (R. 10b), no en la que se suele tocar hoy en día (la perteneciente al Segundo Año de Peregrinaje). 


\begin{tabular}{|c|c|c|c|}
\hline Fecha & Pianos & Mecánica & Repertorio \\
\hline $26-3$ a & Sin determinar & Sin determinar & $\begin{array}{l}\text { Acompañamientos de Lieder (Schubert: Auf dem Wasser zu singen, Die } \\
\text { Taubenpost y Wohin?; Mendelssohn (Auf Flügeln des Gesanges). } \\
\text { Schubert-Liszt: Die Forelle R. 248/61). } \\
\text { Rossini-Liszt: Tarantella R. } 236 . \\
\text { Weber: Aufforderung zum Tanz. }\end{array}$ \\
\hline $26-3 b$ & Sin determinar & Sin determinar & $\begin{array}{l}\text { Acompañamientos de Lieder (Schubert: Der Schiffer, Liszt: Die Vatergnuft R. 601). } \\
\text { Schubert-Liszt: Ständchen R. 243/9. }\end{array}$ \\
\hline $27-3$ & Erard & Inglesa & $\begin{array}{l}\text { Weber-Liszt: Ouvertüre zu Oberon R. } 288 . \\
\text { Schubert-Liszt: una de las Mélodies hongroises R. } 250 . \\
\text { Pacini-Liszt: Divertissement sur la cavatine 'I tuoi frequenti palpiti' R. } 230 . \\
\text { Schachner: Les tourments y La persuasion, Beethoven: } 32 \text { Variaciones en Do } \\
\text { menor. } \\
\text { Improvisación sobre temas dados por el público. }\end{array}$ \\
\hline $28-3$ & Bösendorfer & Inglesa & $\begin{array}{l}\text { Bellini-Liszt: Réminiscences de Norma R. } 133 . \\
\text { Kullak: Paraphrase du quatrième acte de l'Opéra Dom Sébastien de Kullak. } \\
\text { Schubert-Liszt: Aires húngaros (quizás R. 105d). }\end{array}$ \\
\hline $29-3$ & Sin determinar & Sin determinar & Acompañamiento de Lied (Beethoven: An die ferne Geliebte). \\
\hline $31-3$ & Bösendorfer & Inglesa & $\begin{array}{l}\text { Beethoven: Sonata op. } 110 . \\
\text { Döhler: Mélodies russes op. } 60 . \\
\text { Lickl: romanza de Lickl. } \\
\text { Liszt: una fantasía llamada por Adami Réminiscences d'Espagne (pro- } \\
\text { bablemente R. 89) y las Réminiscences de "Don Juan" R. } 228 .\end{array}$ \\
\hline $4-4$ & Streicher & Vienesa (?) & $\begin{array}{l}\text { Weber: Ouvertüre zu Oberon R.288. Acompañamiento de Lied } \\
\text { (Beethoven: Adélaïde). } \\
\text { Meyerbeer-Liszt: Réminiscences de Robert le diable R. } 222 . \\
\text { Haendel: Andante y variaciones en Mi mayor }{ }^{68} \text {. } \\
\text { Schubert: Marcha de Schubert. } \\
\text { Bellini-Liszt: Fantaisie sur des motifs favoris de l'opéra La sonnambula R. } \\
\text { 132. } \\
\text { Weber: Aufförderung zum Tanz. } \\
\text { Schubert-Liszt: Aires húngaros }{ }^{69} \text {. }\end{array}$ \\
\hline \multirow[t]{2}{*}{$17-5$} & Erard & Inglesa & $\begin{array}{l}\text { Beethoven: Concierto en Mi bemol mayor. } \\
\text { Weber: Konzertstück. } \\
\text { Czerny: Grandes variations di bravura. }\end{array}$ \\
\hline & Streicher & Vienesa (?) & $\begin{array}{l}\text { Schubert-Liszt: Mélodies hongroises (R. 250). } \\
\text { Auber: Tarantelle di bravura d'après la tarantelle de La Muette de Portici } \\
\text { R. } 117 \text {. } \\
\text { Acompañamientos de Lieder (Liszt: Il m'aimait tant R. } 566 \text { y Com- } \\
\text { ment, disaient-ils R. } 570 \text { a). } \\
\text { Acompañamientos de ópera (Weber; aria del Oberon; Mozart: aria de } \\
\text { Las bodas de Fígaro). }\end{array}$ \\
\hline
\end{tabular}

${ }^{66}$ Quizás R. 105d, como ya se ha apuntado con anterioridad.

${ }^{67}$ Probablemente, la op. 110.

${ }^{68}$ Probablemente, las conocidas como El herrero armonioso.

${ }^{69}$ Recuérdese que, tal como ya se ha dicho más arriba, esta obra la identifica Legány como R. 105d. 


\section{Conclusiones}

En el momento actual, en que el interés por la práctica interpretativa histórica está en auge, resulta cada vez más urgente conocer los detalles acerca de las herramientas empleadas por el compositor para concebir las obras y para transmitirlas al público, ya que ello proporciona una información muy realista sobre el ideal sonoro que él tenía in mente al crear su música.

La atención que los críticos prestaban a los instrumentos indica que había una gran curiosidad por ese factor. Hoy en día es extraño que se hable del piano de una actuación pública -salvo que tenga algo muy llamativo e inusual, como el nuevo modelo de encordadura recta que usa en tiempos recientes Barenboim-, pero en aquella época de auténtica efervescencia constructiva las cosas eran diferentes: no solo había expectación por lo que hacía el pianista, sino también por lo que el instrumento era capaz de producir y aguantar. El fenómeno es comparable a lo que sucede en competiciones deportivas como la Fórmula 1, donde tan relevante es el piloto como el coche que conduce.

El examen de los documentos ha permitido establecer que Liszt tocó en sus conciertos de Viena de 1838, 1839, 1840 y 1846 pianos de Erard, Graf, Streicher, Bösendorfer y Carl Stein. De ellos, los utilizados en más ocasiones fueron los de Conrad Graf, seguidos por los Streicher. A pesar de tener instrumentos de Erard a su disposición, el maestro húngaro solo los empleó en cuatro conciertos. Las críticas identifican dos casos en los que fabricantes vieneses presentaron pianos provistos no de la mecánica habitual en esa región europea, sino de la inglesa (Streicher y Bösendorfer). Por otro lado, Liszt dispuso, al menos en cinco ocasiones, de más de un piano en el escenario; en tres de esos eventos, se usaron consecutivamente modelos de mecánicas distintas.

Cuadro 5. Fabricantes y número de eventos

\begin{tabular}{|l|l|l|}
\hline Fabricante & Número de eventos y año & Mecánica \\
\hline \multirow{2}{*}{ Erard } & $2(1838)$ & \multirow{2}{*}{ Inglesa } \\
\cline { 1 - 2 } & $2(1846)$ & \\
\hline & $8(1838)$ & \multirow{2}{*}{ Vienesa } \\
\cline { 1 - 2 } & $3(1839)$ & \\
\cline { 1 - 2 } & $4(1840)$ & \multirow{2}{*}{ Vienesa (un ejemplar con inglesa) } \\
\hline Streicher & $7(1846)$ & Vienesa (un ejemplar con inglesa) \\
\hline Bösendorfer & $3(1846)$ & Vienesa \\
\hline Carl Stein & $1(1846)$ & Sin determinar \\
\hline Sin determinar & $4(1838)$ & \\
\cline { 2 - 2 } & $5(1846)$ & \\
\hline
\end{tabular}


Según se desprende de los textos, los pianos de esa época daban bastantes problemas en el escenario. Los críticos parecían sorprenderse cuando alguno de ellos conseguía salir airoso de lo que consideraban una verdadera prueba de fuego: resistir a las manos de Liszt y a un repertorio tan exigente desde un punto de vista mecánico. Los fallos que se solían producir eran la rotura de cuerdas y la poca estabilidad de la afinación. En varios casos, se les achacaron los percances no solo a la energía con la que tocaba Liszt, sino al calor reinante en la sala (como se ha explicado ya, dicha temperatura iba aparejada a un aumento grande de la humedad ambiente). Los instrumentos de Erard, Graf y Streicher adolecieron en varias ocasiones de los desajustes mencionados. El de Carl Stein solo se empleó una vez y el comentario acerca de su respuesta fue positivo. Los Bösendorfer se comportaron de manera impecable en todos los aspectos. En lo referente a la calidad de sonido, las observaciones reflejan siempre una buena impresión. Se valoraba la presencia de una sonoridad plena y potente, concepto que debe entenderse hoy en día teniendo en cuenta que los pianos de aquel momento producían un timbre muy delgado, incisivo y hasta agrio, a causa del recubrimiento de cuero de unos macillos de tamaño mucho menor que los actuales. De ningún modo debe identificarse el sonido lleno del que hablan los textos con el dulce y acampanado del piano moderno.

En sus artículos, los críticos son más benevolentes con los pianos de constructores locales que con el foráneo Erard. Hay que considerar la posibilidad de que sus observaciones no sean del todo imparciales, sino que con ellas estén tratando de apoyar a la industria vienesa frente al producto extranjero.

Como en la mayoría de los conciertos Liszt solo disponía de instrumentos de un mismo tipo de mecánica, la única manera de saber si escogía un repertorio específico para cada uno de ellos es observar los tres casos en los que sí coincidieron pianos vieneses e ingleses en el escenario. En el primero de ellos, el del 18 de abril de 1838, utilizó el Erard para tocar una obra de piano y orquesta, mientras que las obras de bravura y el acompañamiento de un Lied los hizo en los dos Graf. En el segundo, del 1 de marzo de 1846, solo empleó el Streicher de mecánica inglesa, en el cual interpretó transcripciones de Schubert, una sonata de Beethoven y piezas de bravura propias; es posible que el otro piano que había en el escenario, del mismo fabricante, estuviera dotado de mecánica vienesa, pero Adami no lo dice expresamente, de modo que no se puede afirmar con seguridad que ahí el compositor prefiriera un tipo de mecanismo frente a otro. En el tercero de los conciertos mencionados, que tuvo lugar el 17 de mayo de 1846, abordó las obras de piano y orquesta en el Erard, mientras que las de bravura y una transcripción de Schubert las ejecutó en el de mecánica vienesa. Aunque la muestra es pequeña para poder establecer una relación clara entre mecánicas y clase de repertorio, sí pa- 
rece que Liszt daba preferencia a la inglesa, que tenía un mayor poderío dinámico y una gran capacidad de crear masas sonoras, para enfrentarse a una orquesta como solista. La claridad, transparencia y agilidad de la vienesa, en cambio, le resultaban más favorables para las obras brillantes y para la escritura densamente polifónica de las transcripciones. En cualquier caso, los documentos prueban que, aunque el maestro húngaro se adaptaba bien a lo que hubiese en el escenario en ese momento, manejando pianos bastante distintos sin mayor problema, sí que se decantaba por los vieneses frente al Erard, tal y como se deduce del hecho de que hubiese escogido estos en numerosos casos, a pesar de haber tenido a su disposición ejemplares del fabricante francés. No obstante, hay que considerar la posibilidad de que unos factores importantes para su elección fueran, por un lado, apoyar la industria local del piano y, por otro, ofrecer al público y a la crítica un tipo de sonido que les resultara familiar.

Todo lo explicado en el párrafo anterior pone de relieve para el intérprete actual de instrumento histórico que puede servirse de pianos de diferentes mecánicas para tocar Liszt, sin tener miedo a utilizar los vieneses para piezas de escritura muy complicada. El mismo maestro ejecutaba obras de una gran exigencia técnica -como la versión de 1838 de varios de sus estudios o fantasías diversas sobre temas operísticos- sin problemas en estos últimos, prefiriéndolos incluso a los más robustos ingleses. El gusto de Liszt por los pianos de sonoridad clara es una guía también para el artista que toque ese repertorio en el instrumento moderno, el cual ha de buscar -si desea realizar una interpretación acorde con la idea de este compositorobtener no un volumen atronador y una textura gruesa y borrosa, sino un efecto chispeante, transparente, ágil y preciso que se acerque lo más posible al del medio original. 